PROCEEDINGS OF THE

AMERICAN MATHEMATICAL SOCIETY

Volume 135, Number 6, June 2007, Pages 1741-1752

S 0002-9939(07)08658-3

Article electronically published on January 31,2007

\title{
CHAOS FOR POWER SERIES OF BACKWARD SHIFT OPERATORS
}

\author{
FÉLIX MARTÍNEZ-GIMÉNEZ
}

(Communicated by Joseph A. Ball)

\begin{abstract}
We study when the operator $f\left(B_{w}\right)$ is chaotic in the sense of Devaney on a Köthe echelon sequence space, where $B_{w}$ is a weighted backward shift and $f(z)=\sum_{j=0}^{\infty} f_{j} z^{j}$ is a formal power series.
\end{abstract}

\section{INTRODUCTION AND PRELIMINARIES}

A continuous and linear operator $T: E \rightarrow E$ is said to be hypercyclic provided there exist a vector $x \in E$ whose orbit $\operatorname{Orb}(T, x):=\left\{x, T x, T^{2} x, \ldots\right\}$ is dense in $E$. In this case the vector $x$ is said to be a hypercyclic vector for $T$. The article [15] of Grosse-Erdmann is an excellent survey for hypercyclic operators until 1999, check also [17] for more up-to-date results.

Following Devaney [12, a continuous map $T: X \rightarrow X$ on a metric space $X$ is called chaotic if (1) $T$ is topologically transitive (that is, for every pair of nonempty open subsets $U, V$ in $X$ there is $n \in \mathbb{N}$ such that $T^{n}(U) \cap V \neq \emptyset$ ), (2) $T$ has a dense set of periodic points, and (3) $T$ possesses a certain 'sensitivity to initial conditions'. Several authors (see, e.g. 1]) proved that sensitivity to initial conditions is redundant in Devaney's definition. When $X$ is separable, complete and has no isolated points, transitivity is equivalent to the existence of a dense orbit. Moreover, Godefroy and Shapiro [14] showed that hypercyclic operators have sensitive dependence on initial conditions. In other words, hypercyclic operators with a dense set of periodic points are chaotic. We refer to [14, Sec. 6] for the fundamentals of chaotic linear operators and to 15 and 8 for more recent results.

Our framework will be linear and continuous operators $T: E \rightarrow E$ on separable Fréchet spaces $E$, i.e. vector spaces $E$ which have an increasing sequence $\left\{\|\cdot\|_{n}\right\}_{n \geq 1}$ of seminorms that define a metric, which is invariant by addition,

$$
d(x, y):=\sum_{n=1}^{\infty} \frac{1}{2^{n}} \frac{\|x-y\|_{n}}{1+\|x-y\|_{n}}, \quad x, y \in E,
$$

under which $E$ is complete and separable. In what follows $f(z)=\sum_{j=1}^{\infty} f_{j} z^{j}$ is a formal power series, and we are interested in the chaotic behavior of $f(R)$, under

Received by the editors March 14, 2005 and, in revised form, January 20, 2006.

2000 Mathematics Subject Classification. Primary 47A16, 47B37, 37D45, 46A04, 46A45.

Key words and phrases. Chaotic operators, hypercyclic operators, weighted backward shifts.

This work was supported by FEDER and MCYT, Proyecto No. MTM2004-02262 and AVCIT Grupo 03/050.

(C)2007 American Mathematical Society Reverts to public domain 28 years from publication 
the assumption that $f(R)$ is a continuous and well-defined operator on a Köthe sequence space and $R: E \rightarrow E$ is a (weighted) backward shift.

To establish the chaotic behavior of operators we use the following result, which is based on an abundant supply of eigenvectors.

Proposition 1.1 (Eigenvalue Criterion for Chaos 8]). Let $T: E \rightarrow E$ be an operator. If the spaces

$$
\begin{aligned}
X & :=\operatorname{span}\{x \in E / T x=\lambda x,|\lambda|<1\}, \\
Y & :=\operatorname{span}\{y \in E / T y=\lambda y,|\lambda|>1\}, \\
Z & :=\operatorname{span}\left\{z \in E / T z=\lambda z,|\lambda|=1, \lambda \text { is an } n^{\text {th }}{ }_{\text {-root }} \text { of } 1\right\}
\end{aligned}
$$

are dense in $E$, then $T$ is chaotic.

The sets $X$ and $Y$ provide the hypercyclicity of $T$, while $Z$ provides the density of periodic points. This criterion is based on the so-called Hypercyclicity Criterion. It was first discovered by Kitai [19 in her unpublished Ph.D. thesis and rediscovered later by Gethner and Shapiro [13]. J. Bès [5] was able to weaken the conditions (see 23] for a unification of all hypercyclic criteria). Although an eigenvalue criterion for hypercyclicity was formally stated by Bernal in [4, it should be observed that this technique of proving the hypercyclic and chaotic behavior of operators using an abundant supply of eigenvalues was first used by Godefroy and Shapiro [14.

The study of hypercyclic operators on sequence spaces started in 1969 when Rolewicz [24] showed that the weighted backward shift $\lambda B: l_{p} \rightarrow l_{p},\left(x_{1}, x_{2}, \ldots\right) \mapsto$ $\left(\lambda x_{2}, \lambda x_{3}, \ldots\right)$ is hypercyclic if $|\lambda|>1$. He was much interested in the study of orbits of elements in order to answer several operator theory questions and problems. Since then weighted backward shift operators defined on sequence spaces have emerged as a natural and appropriate setting for testing hypercyclicity; see, for example, [14, 25, 21, 9, 16, 11]. H.N. Salas [25] characterized those weighted shifts (unilateral and bilateral) which are hypercyclic.

Rolewicz's example was not the starting point for hypercyclicity. Forty years before, G.D. Birkhoff [6] proved the existence of a 'universal' entire function such that its translates approximate any other entire function as much as desired on compact sets. In other words, he showed that the translation operator $T_{a}: \mathcal{H}(\mathbb{C}) \rightarrow$ $\mathcal{H}(\mathbb{C}), f(z) \mapsto f(z+a),(a \neq 0)$ is hypercyclic on the Fréchet space $\mathcal{H}(\mathbb{C})$ of entire functions endowed with the topology of uniform convergence on compact sets. Later on, MacLane [20] proved the hypercyclicity of the differentiation operator defined on the same space.

Certain operators on function spaces may be represented as weighted backward shift operators on Köthe echelon sequence spaces. Characterizations for hypercyclicity and chaos of such shift operators can be found in 21] (see [16] for more general sequence spaces and operators). MacLane's example can be represented as a weighted backward shift on a Köthe echelon space (see Example 4.4 for the details). The differentiation operator acting on other spaces of entire functions also admits such representation, but with a different sequence space (see e.g. [10] for Hilbert spaces of entire functions and [7] for more general spaces of entire functions). Chan and Shapiro studied in [10] the hypercyclicity of the translation operator on the Hilbert space of entire functions $E^{2}(\gamma)$ (see Example 4.3 for the definition of this space). They observed that $T_{a}=f(D)$ where $f(z)=e^{a z}$, and they also asked 
about the hypercyclicity of other operators. J.H. Shapiro 26] characterized simple connectivity for domains $G$ of the complex plane by dynamical properties of certain linear differential operators acting on $\mathcal{H}(G)$. To be precise, he proved that $G$ is simply connected if and only if $p(D)$ is hypercyclic on $\mathcal{H}(G)$ if and only if $p(D)$ is chaotic on $\mathcal{H}(G)$, where $p$ is a non-constant polynomial. DeLaubenfels and Emamirad characterized chaos for holomorphic functions of shift operators on weighted $l_{p}$ spaces in [11. Operators $T$ such that $f(T)$ is hypercyclic are also studied in [18, 3].

The remainder of this section is devoted to establishing some more necessary notation. In section 2 we study conditions under which power series of (non-weighted) backward shifts are chaotic. Finally we deal whith the weighted case in section 3

Our notation for Köthe echelon spaces and Fréchet spaces is standard, and we refer to [22]. An infinite matrix $A=\left(a_{i, k}\right)_{i, k \in \mathbb{N}}$ is called a Köthe matrix if, for each $i \in \mathbb{N}$, there exists a $k \in \mathbb{N}$ with $a_{i, k}>0$, and $0 \leq a_{i, k} \leq a_{i, k+1}$ for all $i, k \in \mathbb{N}$. For $1 \leq p<\infty$, we consider the (separable) Fréchet spaces

$$
\lambda_{p}(A):=\left\{x \in \mathbb{C}^{\mathbb{N}}:\|x\|_{k}:=\left(\sum_{i=1}^{\infty}\left|x_{i} a_{i, k}\right|^{p}\right)^{1 / p}<\infty, \forall k \in \mathbb{N}\right\},
$$

and for $p=0$

$$
\lambda_{0}(A):=\left\{x \in \mathbb{C}^{\mathbb{N}}: \lim _{i \rightarrow \infty} x_{i} a_{i, k}=0,\|x\|_{k}:=\sup _{i \in \mathbb{N}}\left|x_{i}\right| a_{i, k}, \forall k \in \mathbb{N}\right\},
$$

which are the corresponding Köthe echelon spaces. The latter space is denoted by $c_{0}(A)$ in 22. Examples of classical sequences spaces for concrete Köthe matrices are shown at the end of section 3 .

Given a sequence $\left\{w_{i}\right\}_{i \geq 2}$ of complex numbers (called a weight sequence in the sequel), we define the associated weighted backward shift $B_{w}$ as

$$
B_{w}\left(x_{1}, x_{2}, x_{3}, \ldots\right):=\left(w_{2} x_{2}, w_{3} x_{3}, w_{4} x_{4}, \ldots\right) .
$$

Given a formal power series $f(z)=\sum_{j=0}^{\infty} f_{j} z^{j}$, to define $f\left(B_{w}\right)$ we will use the obvious way of substituting $z$ by the backward shift $B_{w}$. To ensure that this power series converges we need extra conditions on the weights, the Köthe matrix, and the series coefficients that will be stated in the next proposition. For the condition involved we make the convention that in case $a_{i+j, m}=0$ we have $a_{i, n}=0$ or $f_{j}=0$ and we consider $0 / 0$ as 1 . Its proof also defines the operator $f\left(B_{w}\right)$.

Proposition 1.2 (Definition of $f\left(B_{w}\right)$ ). Let $A$ be a Köthe matrix, let $\left\{w_{i}\right\}_{i>2}$ be a weight sequence and let $f(z)=\sum_{j=0}^{\infty} f_{j} z^{j}$ be a power series. If $A,\left\{w_{i}\right\}_{i \geq 2}$ and $f(z)$ satisfy the condition

$$
\forall n \in \mathbb{N}, \exists m>n: \sum_{f_{j} \neq 0}\left|f_{j}\right| \sup _{i \in \mathbb{N}}\left|\prod_{r=1}^{j} w_{i+r}\right|\left(\frac{a_{i, n}}{a_{i+j, m}}\right)<\infty,
$$

then $f\left(B_{w}\right)$ is a continuous and well-defined operator on $\lambda_{p}(A)$ for $1 \leq p<\infty$ or $p=0$.

Proof. Fix $n \in \mathbb{N}$. By (1.1) there exists $m>n$ such that

$$
\sum_{f_{j} \neq 0}\left|f_{j}\right| M_{j}:=\sum_{f_{j} \neq 0}\left|f_{j}\right| \sup _{i \in \mathbb{N}}\left|\prod_{r=1}^{j} w_{i+r}\right|\left(\frac{a_{i, n}}{a_{i+j, m}}\right)<\infty,
$$


where for $f_{j} \neq 0$ we set $M_{j}:=\sup _{i \in \mathbb{N}}\left|\prod_{r=1}^{j} w_{i+r}\right|\left(\frac{a_{i, n}}{a_{i+j, m}}\right)$. For $x \in \lambda_{p}(A)$ and $j \in \mathbb{N}$ with $f_{j} \neq 0$ we have

$$
\begin{aligned}
\left\|f_{j} B_{w}^{j} x\right\|_{n}^{p} & =\left|f_{j}\right|^{p}\left(\sum_{i=1}^{\infty}\left|\left(\prod_{r=1}^{j} w_{i+r}\right) x_{i+j} a_{i, n}\right|^{p}\right) \\
& \leq\left|f_{j}\right|^{p}\left(\sum_{i=1}^{\infty} M_{j}^{p}\left|x_{i+j} a_{i+j, m}\right|^{p}\right) \leq\left|f_{j}\right|^{p} M_{j}^{p}\|x\|_{m}^{p},
\end{aligned}
$$

hence $\left\|f_{j} B_{w}^{j} x\right\|_{n} \leq\left|f_{j}\right| M_{j}\|x\|_{m}$ for all $x \in \lambda_{p}(A)$ and all $j \in \mathbb{N}$ with $f_{j} \neq 0$. This implies

$$
\sum_{f_{j} \neq 0}\left\|f_{j} B_{j} x\right\|_{n} \leq\|x\|_{m} \sum_{f_{j} \neq 0}\left|f_{j}\right| M_{j}<\infty,
$$

and it follows that $\sum_{f_{j} \neq 0} f_{j} B^{j}$ converges pointwise on $\lambda_{p}(A)$. We denote the limit operator by $f\left(B_{w}\right)$. The Banach-Steinhaus Theorem gives the conclusion. For $p=0$ the proof is similar to the supremum norm.

Remark 1.3. (1) Condition (1.1) is sufficient to have $f\left(B_{w}\right)$ continuous and well defined on $\lambda_{p}(A)$. If we look further and we ask for necessary and sufficient conditions, no clear answer is available. To understand that, consider first, for each $k \in \mathbb{N}$, the weighted Banach spaces $l_{p}\left(\left(a_{i, k}\right)_{i}\right):=\left\{\left(x_{i}\right)_{i} \in \mathbb{C}^{\mathbb{N}}:\left(x_{i} a_{i, k}\right)_{i} \in l_{p}\right\}$. It is clear that $f\left(B_{w}\right)$ is continuous and well defined on $\lambda_{p}(A)$ if and only if for each $n \in \mathbb{N}$ there exists $m>n$ such that $f\left(B_{w}\right): l_{p}\left(\left(a_{i, m}\right)_{i}\right) \rightarrow l_{p}\left(\left(a_{i, n}\right)_{i}\right)$ is continuous and well defined. Even more, considering the natural isometry $\phi_{k}: l_{p}\left(\left(a_{i, k}\right)_{i}\right) \rightarrow l_{p}$, $\left(x_{i}\right)_{i} \mapsto\left(x_{i} a_{i, k}\right)_{i}$, we find out that we need to use 'computable' conditions in terms of the entries of a matrix of the operator $\phi_{n} \circ f\left(B_{w}\right) \circ \phi_{m}^{-1}: l_{p} \rightarrow l_{p}$. Namely, the matrix $\left(t_{i j}\right)$ of $\phi_{n} \circ f\left(B_{w}\right) \circ \phi_{m}^{-1}$ respect to the canonical basis is an upper triangular matrix with entries $t_{i j}=\left(a_{i, n} / a_{j, m}\right) f_{j-i} \prod_{r=i+1}^{j} w_{r}$ if $i \leq j$ and 0 otherwise.

The problem of characterizing bounded operators on $l_{p}$ in terms of the (canonical) matrix operator is studied, in the general case, in [27, Chapter 8], and it is completely solved for $p=0$ and $p=1$. For $p=0$ we have that an operator, with canonical matrix $\left(t_{i j}\right)$, defined on $c_{0}$ is bounded if and only if $\sup _{i} \sum_{j}\left|t_{i j}\right|<\infty$ and for each $j \in \mathbb{N}$ the sequence $\left(t_{i j}\right)_{i} \in c_{0}$ (see [27, 8.4.5A]). In our case, taking into account that the matrix operator is upper triangular, we have that $f\left(B_{w}\right): \lambda_{0}(A) \rightarrow \lambda_{0}(A)$ is continuous and well defined if and only if

$$
\forall n \in \mathbb{N}, \exists m>n: \sup _{i \in \mathbb{N}} \sum_{j=i}^{\infty} \frac{a_{i, n}}{a_{j, m}}\left|f_{j-i} \prod_{r=i+1}^{j} w_{r}\right|<\infty .
$$

For $p=1$ we have that an operator defined on $l_{1}$ is bounded if and only if $\sup _{j} \sum_{i}\left|t_{i j}\right|<\infty$ (see [27, 8.4.1D]). In our case, again observing the triangular structure of the matrix, we have that $f\left(B_{w}\right): \lambda_{1}(A) \rightarrow \lambda_{1}(A)$ is continuous and well defined if and only if

$$
\forall n \in \mathbb{N}, \exists m>n: \sup _{j \in \mathbb{N}} \sum_{i=1}^{j} \frac{a_{i, n}}{a_{j, m}}\left|f_{j-i} \prod_{r=i+1}^{j} w_{r}\right|<\infty .
$$

For $1<p<\infty$ no tidy characterizations of bounded operators on $l_{p}$ are known in terms of $\left(t_{i j}\right)$. Using Hölder's inequality it is an easy exercise to prove that if each row and each column of the matrix is absolutely summable with all the 
sums uniformly bounded, that is, $\max \left\{\sup _{i} \sum_{j}\left|t_{i j}\right|, \sup _{j} \sum_{i}\left|t_{i j}\right|\right\}<\infty$, then the corresponding operator is bounded on $l_{p}$ (see also [27, 8.5.12]). This sufficient condition is clearly fulfilled in our case if (1.2) and (1.3) are both satisfied. Thus (1.2) and (1.3) imply that $f\left(B_{w}\right): \lambda_{p}(A) \longrightarrow \lambda_{p}(A)$ is continuous and well defined for $1<p<\infty$. For simplicity and in order to handle $1 \leq p<\infty$ and $p=0$ at once, we will use condition (1.1) for the remainder of this paper.

(2) Special interest has the case when the space $\lambda_{p}(A)$ is nuclear (this includes many natural examples such as $\mathcal{H}(\mathbb{C}), \mathcal{H}(\mathbb{D})$, the space of rapidly decreasing sequences, etc.). In this case we have that $\lambda_{p}(A)=\lambda_{q}(A)=\lambda_{0}(A)$ for all $p, q$ with $1 \leq p, q \leq \infty$ (see [22, Prop. 28.16]) and any of the conditions we have seen so far may be used. Moreover, conditions (1.2) and (1.3) are characterizations to have $f\left(B_{w}\right)$ continuous and well defined on $\lambda_{p}(A)$.

\section{The (NON-Weighted) CASE $f(B)$}

For this section we assume $\left\{w_{i}\right\}_{i \geq 2}=\{1\}_{i \geq 2}$; its associated backward shift is denoted as $B$. In this case condition (1.1) takes the form

$$
\forall n \in \mathbb{N}, \exists m>n: \sum_{f_{j} \neq 0}\left|f_{j}\right| \sup _{i \in \mathbb{N}}\left(\frac{a_{i, n}}{a_{i+j, m}}\right)<\infty .
$$

Remark 2.1. The arguments we use to prove the hypercyclicity of $f(B)$ are based on an abundant supply of eigenvectors of $B$ and $f(B)$. Let us be more precise: In what follows the topology of the sequence space is not used. We consider a power series $f(z)=\sum_{j=0}^{\infty} f_{j} z^{j}$, and we assume that the backward shift $B\left(x_{1}, x_{2}, \ldots\right):=$ $\left(x_{2}, x_{3}, \ldots\right)$ and the linear map $f(B)\left(x_{1}, x_{2}, \ldots\right):=\sum_{j=0}^{\infty} f_{j} B^{j}\left(x_{1}, x_{2}, \ldots\right)$ are well defined on the underlying sequence space.

We have that $x=\left(x_{i}\right)_{i \geq 1}$ is an eigenvector of $B$ with eigenvalue $\lambda \in \mathbb{C}$ if $x=\left(\lambda^{i}\right)_{i \geq 1}$. Moreover, $x$ is also an eigenvector of $f(B)$ with eigenvalue $f(\lambda)$, that is, $f(B) x=f(\lambda) x$.

The eigenvectors just mentioned will play an important role when applying the Eigenvalue Criterion for Chaos. The density of these eigenvectors is also a necessary ingredient that is ensured by the following density lemma.

Lemma 2.2. Let $A$ be a Köthe matrix and let $1 \leq p<\infty$ or $p=0$. Let $U \subset \mathbb{C}$ be an open set such that $\left\{\left(z^{i}\right)_{i \geq 1}: z \in U\right\} \subset \lambda_{p}(A)$. If $C \subset U$ has a limit point, then $\operatorname{span}\left\{\left(z^{i}\right)_{i \geq 1}: z \in C\right\}$ is dense in $\lambda_{p}(A)$. In particular, if $U$ in non-empty, then $\operatorname{span}\left\{\left(z^{i}\right)_{i \geq 1}: z \in U\right\}$ is dense in $\lambda_{p}(A)$.

Proof. We set $X:=\operatorname{span}\left\{\left(z^{i}\right)_{i \geq 1}: z \in C\right\}$. By the Hahn-Banach Theorem it is enough to see that if $y \in \lambda_{p}^{\prime}(A)$ vanishes on $X$, then $y=0$. We fix $y \in \lambda_{p}^{\prime}(A)$ such that

$$
\langle x, y\rangle=\sum_{i=1}^{\infty} x_{i} y_{i}=0, \forall x \in X .
$$

We define $g(z):=\sum_{i=1}^{\infty} y_{i} z^{i}$, which is a holomorphic function on $U$. For $z \in C$ we have

$$
g(z)=\sum_{i=1}^{\infty} y_{i} z^{i}=\left\langle\left(z^{i}\right)_{i \geq 1}, y\right\rangle=0 .
$$

Since $C$ has a limit point and $g(z)$ is holomorphic on $U$, we obtain $g=0$, which implies that $y_{i}=0$ for all $i \in \mathbb{N}$ and then $y=0$. 
Theorem 2.3. Let $f(z)$ be a power series and let $A$ be a Köthe matrix satisfying condition (2.1). If

$$
\sup _{k \in \mathbb{N}}\left(\limsup _{i \in \mathbb{N}} a_{i, k}^{1 / i}\right)=: M<\infty
$$

and $f(z)$ defines a non-constant holomorphic function on $\frac{1}{M} \mathbb{D}$ with $f\left(\frac{1}{M} \mathbb{D}\right) \cap \partial \mathbb{D} \neq \emptyset$ $\left(\frac{1}{M} \mathbb{D}:=\mathbb{C}\right.$ if $\left.M=0\right)$, then $f(B)$ is chaotic on $\lambda_{p}(A), 1 \leq p<\infty$ or $p=0$.

Proof. The condition on the matrix $A$ implies that $\left\{\left(\lambda^{i}\right)_{i \geq 1}: \lambda \in M^{-1} \mathbb{D}\right\} \subset \lambda_{p}(A)$. On the other hand, since $f(z)$ is a non-constant holomorphic function on $M^{-1} \mathbb{D}$ (hence it is open) and intersects the unit circle, we have that

$$
\begin{aligned}
& B:=\left\{\lambda \in M^{-1} \mathbb{D}:|f(\lambda)|<1\right\}=f^{-1}(\mathbb{D}) \cap M^{-1} \mathbb{D} \text { and } \\
& C:=\left\{\lambda \in M^{-1} \mathbb{D}:|f(\lambda)|>1\right\}=f^{-1}(\mathbb{C} \backslash \overline{\mathbb{D}}) \cap M^{-1} \mathbb{D}
\end{aligned}
$$

are both non-empty open sets. We take

$$
X:=\operatorname{span}\left\{\left(\lambda^{i}\right)_{i \geq 1}: \lambda \in B\right\} \text { and } Y:=\operatorname{span}\left\{\left(\lambda^{i}\right)_{i \geq 1}: \lambda \in C\right\},
$$

which are dense in $\lambda_{p}(A)$ by Lemma 2.2 .

Next, since $f\left(\frac{1}{M} \mathbb{D}\right) \cap \partial \mathbb{D} \neq \emptyset$ we have that $f\left(\frac{1}{M} \mathbb{D}\right)$ contains an arc of the unit circle $\partial \mathbb{D}$. Let $V$ be the roots of unity that lie in that arc. We define the (nonempty) set $D:=f^{-1}(V) \cap M^{-1} \mathbb{D}$. The density of the roots of unity in the unit circle and, in particular, the density of $V$ in the above-mentioned arc implies that $D$ contains a limit point. Hence the set $Z:=\operatorname{span}\left\{\left(\lambda^{i}\right)_{i \geq 1}: \lambda \in D\right\}$ is dense in $\lambda_{p}(A)$ by Lemma 2.2, and the conclusion follows by Proposition 1.1,

Corollary 2.4. Let $f(z)$ be any non-constant entire function and let $A$ be a Köthe matrix satisfying condition (2.1). If $\lim _{i \in \mathbb{N}} a_{i, k}^{1 / i}=0$ for all $k \in \mathbb{N}$, then $f(B)$ is chaotic on $\lambda_{p}(A), 1 \leq p<\infty$ or $p=0$.

Proof. By the Little Picard Theorem the range of $f(z)$ may omit at most one single point and then $f(\mathbb{C})$ always intersects the unit circle.

Remark 2.5. Although Theorem 2.3 only requires $f(B)$ to be continuous without requiring the continuity of $B$, the usual situation is to study the chaotic behavior of $f(B)$ when we have a continuous backward shift $B$. When this is the case an additional condition on the Köthe matrix must be imposed to ensure that $B$ is continuous. It is clear from (2.1) that this condition is

$$
\forall n \in \mathbb{N}, \exists m>n: \sup _{i \in \mathbb{N}} \frac{a_{i, n}}{a_{i+1, m}}<\infty,
$$

which turns out to characterize the continuity of $B$, as an easy computation shows.

At this point it is worth showing the following example. We consider the backward shift $B: l_{p} \rightarrow l_{p}$. The operator $B / 2$ is a contraction, so it cannot be hypercyclic, and hence neither can it be chaotic. On the other hand, if we take $f(z)=1+z$ we obtain that $f(B)=I+B / 2$ is chaotic on $l_{p}$. The next corollary handles the restrictive case when $B$ is chaotic.

Corollary 2.6. Let $f(z)$ be a power series and let $A$ be a Köthe matrix satisfying conditions (2.1) and (2.2). If $B: \lambda_{p}(A) \rightarrow \lambda_{p}(A)$ is chaotic and $f(z)$ defines a non-constant holomorphic function on $\mathbb{D}$ with $f(\mathbb{D}) \cap \partial \mathbb{D} \neq \emptyset$, then $f(B)$ is chaotic on $\lambda_{p}(A), 1 \leq p<\infty$ or $p=0$. 
Proof. By [21, Theorem 3.2] we have that $B$ is chaotic if and only if for each $k \in \mathbb{N}$ $\left\{a_{i, k}\right\}_{i \geq 1} \in l_{p}\left(l_{0}:=c_{0}\right)$. This implies $\lim \sup _{i \in \mathbb{N}} a_{i, k}^{1 / i} \leq 1$ for any $k \in \mathbb{N}$.

\section{The (Weighted) CASe $f\left(B_{w}\right)$}

In this section we study, via commutative diagrams, conditions under which $f\left(B_{w}\right)$ is chaotic. We will use results of the previous section and also the following lemma, whose proof may be found in [21, Lemma 2.1] in a more general form.

Lemma 3.1. Let $T_{i}: E_{i} \rightarrow E_{i}$ be an operator on a separable Fréchet space $E_{i}$, $i=1,2$, and let $\phi: E_{1} \rightarrow E_{2}$ be a homeomorphism such that $T_{2} \circ \phi=\phi \circ T_{1}$, i.e, such that the following diagram commutes:

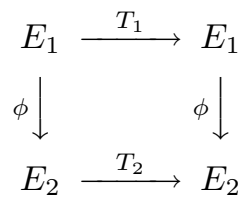

Then $T_{1}$ is chaotic if and only if $T_{2}$ is chaotic.

Given a power series $f(z)$, a Köthe matrix $A$ and a weight sequence $\left\{w_{i}\right\}_{i \geq 2}$ such that condition (1.1) is satisfied and $w_{i} \neq 0$ for all $i \geq 2$ (if $w_{i_{0}}=0$ for some $i_{0} \in \mathbb{N}$, then neither $B_{w}$ nor $f\left(B_{w}\right)$ can be hypercyclic), we put

$$
v_{1}:=1, v_{i}:=\frac{1}{w_{2} \cdots w_{i}}, i>1
$$

We define

$$
\bar{A}:=\left(\bar{a}_{i, k}\right)_{i, k \in \mathbb{N}}: \bar{a}_{i, k}:=\left|v_{i}\right| a_{i, k}, \forall i, k \in \mathbb{N},
$$

and the diagonal transform $\phi_{v}: \lambda_{p}(\bar{A}) \longrightarrow \lambda_{p}(A)$ such that

$$
\phi_{v}\left(x_{1}, x_{2}, x_{3}, \ldots\right):=\left(v_{1} x_{1}, v_{2} x_{2}, v_{3} x_{3}, \ldots\right) .
$$

We may construct the diagram on the right and state the following easy properties (see 21] for the details).

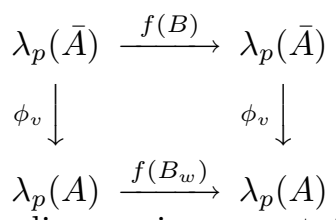

(1) Straightforward calculations show that the above diagram is commutative.

(2) The diagonal transform $\phi_{v}$ is a linear homeomorphism.

(3) By topological conjugacy we have that $f(B)$ is chaotic if and only if $f\left(B_{w}\right)$ is chaotic.

This last property allows us to study the chaotic behavior of $f\left(B_{w}\right)$ on $\lambda_{p}(A)$ from the chaotic behavior of $f(B)$ on $\lambda_{p}(\bar{A})$. The equivalent results for this weighted situation, each one based on the corresponding similar result for the non-weighted case studied in the previous section, are the following.

Theorem 3.2. Let $f(z)$ be a power series, let $\left\{w_{i}\right\}_{i \geq 2}$ be a weight sequence and let $A$ be a Köthe matrix satisfying condition (1.1). If

$$
\sup _{k \in \mathbb{N}}\left(\limsup _{i \in \mathbb{N}} \frac{a_{i, k}^{1 / i}}{\left|\prod_{j=2}^{i} w_{j}\right|^{1 / i}}\right)=: M<\infty,
$$


and $f(z)$ defines a non-constant holomorphic function on $\frac{1}{M} \mathbb{D}$ with $f\left(\frac{1}{M} \mathbb{D}\right) \cap \partial \mathbb{D} \neq \emptyset$ $\left(\frac{1}{M} \mathbb{D}:=\mathbb{C}\right.$ if $\left.M=0\right)$, then $f\left(B_{w}\right)$ is chaotic on $\lambda_{p}(A), 1 \leq p<\infty$ or $p=0$.

Corollary 3.3. Let $f(z)$ be any non-constant entire function, let $\left\{w_{i}\right\}_{i \geq 2}$ be a weight sequence and let $A$ be a Köthe matrix satisfying condition (1.1). If

$$
\lim _{i \in \mathbb{N}} \frac{a_{i, k}^{1 / i}}{\left|\prod_{j=2}^{i} w_{j}\right|^{1 / i}}=0, \forall k \in \mathbb{N},
$$

then $f\left(B_{w}\right)$ is chaotic on $\lambda_{p}(A), 1 \leq p<\infty$ or $p=0$.

A similar observation to Remark 2.5 applies here, that is, $B_{w}$ is continuous in $\lambda_{p}(A)$ if and only if $A$ and $w$ satisfy

$$
\forall n \in \mathbb{N}, \exists m>n: \sup _{i \in \mathbb{N}}\left|w_{i+1}\right| \frac{a_{i, n}}{a_{i+1, m}}<\infty .
$$

Corollary 3.4. Let $f(z)$ be a power series, let $\left\{w_{i}\right\}_{i>2}$ be a weight sequence and let $A$ be a Köthe matrix. Assume that conditions (1.1) and (3.4) are satisfied. If $B_{w}: \lambda_{p}(A) \rightarrow \lambda_{p}(A)$ is chaotic and $f(z)$ defines a non-constant holomorphic function on $\mathbb{D}$ with $f(\mathbb{D}) \cap \partial \mathbb{D} \neq \emptyset$, then $f\left(B_{w}\right)$ is chaotic on $\lambda_{p}(A), 1 \leq p<\infty$ or $p=0$.

\section{EXAMPLES}

Example 4.1. Let $A=(1)_{j, k \in \mathbb{N}}, w=\left\{w_{i}\right\}_{i \geq 2} \in l_{\infty}$ and $1 \leq p<\infty$. Then (1.1) holds for any entire function. If $B_{w}: l_{p} \rightarrow l_{p}$ is chaotic, which is equivalent to $\sum_{i \in \mathbb{N}}\left(\prod_{j=2}^{i}\left|w_{j}^{p}\right|\right)^{-1}<\infty$ (see [21, Example 3.5(1)(ii)]), and $f(z)$ is a non-constant entire function such that $f(\mathbb{D}) \cap \partial \mathbb{D} \neq \emptyset$, then $f\left(B_{w}\right)$ is also chaotic on $l_{p}$. An interesting particular case appears when the weight sequence is constant, that is, $w=\{\lambda\}_{i \geq 2}$. We have $M=1 / \lambda$, and the (Toeplitz!) operator $f(\lambda B)$ is chaotic if $f(\lambda \mathbb{D})$ intersects the unit circle $\partial \mathbb{D}$. It turns out that this condition is actually a characterization for chaos of $f(\lambda B)$ ([11, Theorem 2.3]).

Example 4.2. When the matrix $A$ is such that for each $k \in \mathbb{N}$ there is $i_{k} \in \mathbb{N}$ satisfying $a_{i, k}=0$ for all $i>i_{k}$, then $\lambda_{p}(A)=\omega:=\mathbb{K}^{\mathbb{N}}$, the countable product of the scalar field. Condition (1.1) is satisfied for any polynomial $f(z)$ and $w=\left\{w_{i}\right\}_{i \geq 2}$ arbitrary. In this particular case $B_{w}$ is always chaotic on $\omega$ (see 21, Example $3.5(2)]$ ). Moreover, Corollary 3.3 may be applied, and $f\left(B_{w}\right)$ is chaotic on $\omega$ for any non-constant polynomial $f(z)$.

Example 4.3. Hilbert spaces of entire functions (see [10]). Let $\gamma(z)$ be an admissible comparison entire function, that is, the Taylor coefficients $\gamma_{i}>0$ for all $i \in \mathbb{N}_{0}$ and the sequence $\left\{i \gamma_{i} / \gamma_{i-1}\right\}_{i \geq 1}$ is monotonically decreasing. We consider the Hilbert space $E^{2}(\gamma)$ of power series

$$
g(z)=\sum_{i=0}^{\infty} \hat{g}(i) z^{i}
$$

for which

$$
\|g\|_{2, \gamma}^{2}:=\sum_{i=0}^{\infty} \gamma_{i}^{-2}|\hat{g}(i)|^{2}<\infty
$$


It is clear that $E^{2}(\gamma)$ is isometric to $\lambda_{2}(A)$ with $A=\left(a_{i, k}\right)_{i \in \mathbb{N}_{0}, k \in \mathbb{N}}=\left(\gamma_{i}^{-1}\right)_{i \in \mathbb{N}_{0}, k \in \mathbb{N}}$ and with the identification $f \mapsto\left(f^{(i)}(0) / i \text { ! }\right)_{i \geq 0}$. Moreover, the operator of differentiation turns out to be a weighted backward shift with weights $\left\{w_{i}\right\}_{i \geq 1}=\{i\}_{i \geq 1}$. Since the topology considered in this space is described by a single norm, an easy computation shows that

$$
\sup _{i \in \mathbb{N}}\left|\prod_{r=1}^{j} w_{i+r}\right|\left(\frac{a_{i, n}}{a_{i+j, m}}\right) \leq\left(\frac{\gamma_{1}}{\gamma_{0}}\right)^{j}, \forall j \in \mathbb{N}
$$

and this implies that $f\left(B_{w}\right)$ is continuous for any entire function $f(z)$.

K. Chan and J. Shapiro showed that for $a \neq 0$ the translation operator $T_{a}$ is hypercyclic on $E^{2}(\gamma)$ (see [10, Theorem 2.1]). They proved that $T_{a}=\sum_{n \geq 0} a^{n} / n ! D^{n}$, pointing out that what they showed was the hypercyclicity of $f(D)$ for the particular case $f(z)=e^{a z}$. They also asked for the hypercyclicity of 'other' operators.

Let us now consider only those spaces for which $0<r:=\lim _{i \rightarrow \infty} i \gamma_{i} / \gamma_{i-1}$. The limit of successive roots of the sequence $\left\{i ! \gamma_{i}\right\}_{i \geq 0}$ coincides with the limit of successive ratios $\left\{i \gamma_{i} / \gamma_{i-1}\right\}_{i \geq 1}$. Hence

$$
M:=\sup _{k \in \mathbb{N}}\left(\limsup _{i \in \mathbb{N}} \frac{a_{i, k}^{1 / i}}{\left|\prod_{j=2}^{i} w_{j}\right|^{1 / i}}\right)=\limsup _{i \in \mathbb{N}} \frac{1}{\left(i ! \gamma_{i}\right)^{1 / i}}=\frac{1}{r}<\infty .
$$

By Theorem 3.2 the operator $f\left(B_{w}\right)$ is chaotic (hence hypercyclic) on $\lambda_{2}(A)$ for nonconstant entire functions $f(z)$ such that $f\left(\frac{1}{M} \mathbb{D}\right) \cap \partial \mathbb{D} \neq \emptyset$. This last statement may be rephrased as: For Hilbert spaces $E^{2}(\gamma)$ with admissible comparison functions $\gamma(z)$ satisfying $0<r:=\lim _{i \rightarrow \infty} i \gamma_{i} / \gamma_{i-1}$, the operator $f(D)$ is chaotic (hence hypercyclic) for non-constant entire functions $f(z)$ such that $f(r \mathbb{D}) \cap \partial \mathbb{D} \neq \emptyset$. It should be observed that $E^{2}(\gamma)$ is represented as a weighted $l_{2}$ space, and therefore this example can also be studied from [11.

Example 4.4. Godefroy and Shapiro studied in 14 the hypercyclicity of operators defined on $\mathcal{H}\left(\mathbb{C}^{N}\right)$ that commute with translations. Among others things, they proved that these operators are of the form $f(D)$, where $f(z)$ is an entire function on $\mathbb{C}^{N}$ of exponential type. In this example we show the hypercyclicity and chaos of $f(D)$ using the context of Köthe sequence spaces and weighted shifts.

The space of entire functions with its natural topology (the compact open topology) may be represented as a Köthe echelon space taking

$$
A=\left(a_{i, k}\right)_{i \in \mathbb{N}_{0}, k \in \mathbb{N}}=\left(\frac{e^{i k}}{i !}\right)_{i \in \mathbb{N}_{0}, k \in \mathbb{N}},
$$

and the map $f \stackrel{\Phi}{\longmapsto}\left(f^{(i)}(0)\right)_{i \geq 0}$. It is easy to prove that $\Phi$ is an isomorphism, and we observe that the operator of differentiation is a backward shift with weights $\left\{w_{i}\right\}_{i \geq 1}=\{1\}_{i \geq 1}$, that is, $\Phi \circ D=B \circ \Phi$.

It is not difficult to show that

$$
\sup _{i \in \mathbb{N}_{0}} \frac{a_{i, n}}{a_{i+j, m}} \leq \frac{j^{j}}{e^{j m}} \quad(m>n) .
$$

For entire functions $f(z)$ of exponential type, that is, entire functions for which there exist positive constants $A$ and $B$ such that

$$
|f(z)| \leq A e^{B|z|}, z \in \mathbb{C},
$$


straightforward computations with power series, and the Cauchy inequalities show that the power series coefficients satisfy

$$
\left|f_{j}\right| \leq A \frac{e^{j} B^{j}}{j^{j}}, j \geq 0 .
$$

Therefore

$$
\sum_{f_{j} \neq 0}\left|f_{j}\right| \sup _{i \in \mathbb{N}_{0}} \frac{a_{i, n}}{a_{i+j, m}} \leq A \sum_{j \geq 0} \frac{B^{j}}{e^{j(m-1)}} .
$$

For fixed $n \in \mathbb{N}$, it is enough to choose $m>n$ such that $e^{m-1}>B$ to satisfy condition (2.1). For each $k \in \mathbb{N}$ we have

$$
\lim _{i} a_{i, k}^{1 / i}=\lim _{i} \frac{e^{k}}{i !^{1 / i}}=0 .
$$

Hence by Corollary 2.4 we obtain that $f(B)$ is chaotic on $\lambda_{p}(A)$ for any non-constant entire function $f(z)$ of exponential type. Equivalently, $f(D)$ is chaotic on $\mathcal{H}(\mathbb{C})$ for any non-constant entire function $f(z)$ of exponential type (see also [26] where much more is said about the hypercyclicity and chaos of $f(D)$ on the space of holomorphic functions on domains of the complex plane).

Example 4.5. The Hankel translation operator and the Bessel operator 2. These two operators appear in the theory of the distributional Hankel transform which was used by Zemanian 28, to solve the Dirichlet problem in cylindrical coordinates. Consider the space of even entire functions $\mathcal{H}_{e}(\mathbb{C})$ endowed with the natural compact open topology. Defined on this space we consider the Bessel operator $\Delta_{\mu}=z^{-2 \mu-1} D z^{2 \mu+1} D(\mu>-1 / 2)$.

As in the previous example $\mathcal{H}_{e}(\mathbb{C})$ may be represented as a Köthe echelon space taking $A=\left(a_{i, k}\right)_{i \in \mathbb{N}_{0}, k \in \mathbb{N}}=\left(e^{2 i k} /(2 i) !\right)_{i \in \mathbb{N}_{0}, k \in \mathbb{N}}$, and the map $g \mapsto\left(g^{(2 i)}(0)\right)_{i \geq 0}$. Moreover, if $g(z)=\sum_{j \geq 0} g^{(2 j)}(0) /(2 j) ! z^{2 j} \in \mathcal{H}_{e}(\mathbb{C})$, then

$$
\Delta_{\mu} g(z)=\sum_{j \geq 1} \frac{g^{(2 j)}(0)}{(2 j) !} 4(j+\mu) j z^{2(j-1)}=\sum_{j \geq 0} \frac{g^{(2(j+1))}(0)}{(2 j) !} \frac{j+1+\mu}{j+1 / 2} z^{2 j} .
$$

This implies that the Bessel operator $\Delta_{\mu}$ may be represented as a weighted backward shift with weights $\left\{w_{i}\right\}_{i \geq 1}=\{(i+1+\mu) /(i+1 / 2)\}_{i \geq 1}$. It may be proved that for any entire function $f(z)$ of exponential type $f\left(B_{w}\right)$ is a continuous and well defined operator [2, Prop. 3.5]. By Corollary 3.3 we obtain that $f\left(B_{w}\right)$ is chaotic on $\lambda_{p}(A)$ for any non-constant entire function $f(z)$ of exponential type. Equivalently, $f\left(\Delta_{\mu}\right)$ is chaotic on $\mathcal{H}_{e}(\mathbb{C})$ for any non-constant entire function $f(z)$ of exponential type.

If we take $f(z)=z$ we obtain that the Bessel operator $\Delta_{\mu}$ is chaotic on $\mathcal{H}_{e}(\mathbb{C})$. The Hankel translate of an even entire function $g(z)=\sum_{j \geq 0} g_{2 j} z^{2 j}$ is defined by

$$
\left(\tau_{a}^{\mu} g\right)(z):=\sum_{n=0}^{\infty} g_{2 n} \sum_{k=0}^{n}\left(\begin{array}{l}
n \\
k
\end{array}\right) \frac{\Gamma(n+\mu+1) \Gamma(\mu+1)}{\Gamma(n-k+\mu+1) \Gamma(k+\mu+1)} a^{2 n-k} z^{2 k} .
$$

We take the entire function

$$
f_{a}^{\mu}(z)=\Gamma(\mu+1) \sum_{k=1}^{\infty} \frac{a^{2 k}}{2^{2 k} \Gamma(\mu+k+1) k !} z^{k} .
$$


It turns out that $\tau_{a}^{\mu}=f_{a}^{\mu}\left(\Delta_{\mu}\right)$ (see [2, Prop. 3.5] for the details and also for the proof that $f_{a}^{\mu}(z)$ is of exponential type). In this situation we have that the Hankel translation operator $\tau_{a}^{\mu}$ is chaotic on $\mathcal{H}_{e}(\mathbb{C})$.

\section{ACKNOWLEDGMENTS}

The author wishes to express his thanks to A. Peris for suggesting this research and for several helpful comments concerning the final version of the paper.

\section{REFERENCES}

1. J. Banks, J. Brooks, G. Cairns, G Davis, and P. Stacey, On Devaney's definition of chaos, Amer. Math. Monthly 99 (1992), no. 4, 332-334. MR.1157223 (93d:54059)

2. M. Belhadj and J.J. Betancor, Hankel convolution operators on entire functions and distributions, J. Math. Anal. Appl. 276 (2002), 40-63. MR.1944335 (2003k:46050)

3. T. Bermúdez and V.G. Miller, On operators $T$ such that $f(T)$ is hypercyclic, Integral Equations Operator Theory 37 (2000), no. 3, 332-340. MR1776958 (2001f:47007)

4. L. Bernal-González, Hypercyclic sequences of differential and antidifferential operators, J. Approx. Theory 96 (1999), no. 2, 323-337. MR.1671201 (2000b:47081)

5. J. P. Bès and A. Peris, Hereditarily hypercyclic operators, J. Funct. Anal. 167 (1999), no. 1, 94-112. MR.1710637 (2000f:47012)

6. G.D. Birkhoff, Démonstration d'un théoréme elémentaire sur les fonctions entiéres, C. R. Acad. Sci. Paris 189 (1929), 473-475.

7. J. Bonet, Hypercyclic and chaotic convolution operators, J. London Math. Soc. 62 (2000), no. 1, 253-262. MR1772185 (2001g:47053)

8. J. Bonet, F. Martínez-Giménez, and A. Peris, Linear chaos on Fréchet spaces, Internat. J. Bifur. Chaos Appl. Sci. Engrg. 13 (2003), no. 7, 1649-1655. MR2015614 (2004i:47016)

9. P.S. Bourdon and J.H. Shapiro, Hypercyclic operators that commute with the Bergman backward shift, Trans. Amer. Math. Soc. 352 (2000), no. 11, 5293-5316. MR1778507(2001i:47053)

10. K.C. Chan and J.H. Shapiro, The cyclic behavior of translation operators on Hilbert spaces of entire functions, Indiana Univ. Math. J. 40 (1991), no. 4, 1421-1449. MR1142722 (92m:47060)

11. R. deLaubenfels and H. Emamirad, Chaos for functions of discrete and continuous weighted shift operators, Ergodic Theory Dynam. Systems 21 (2001), no. 5, 1411-1427. MR1855839 (2002j:47030)

12. R.L. Devaney, An introduction to chaotic dynamical systems, 2nd ed., Addison-Wesley, Reading, MA, 1989. MR1046376 (91a:58114)

13. R.M. Gethner and J.H. Shapiro, Universal vectors for operators on spaces of holomorphic functions, Proc. Amer. Math. Soc. 100 (1987), no. 2, 281-288. MR0884467 (88g:47060)

14. G. Godefroy and J.H. Shapiro, Operators with dense, invariant, cyclic vector manifolds, J. Funct. Anal. 98 (1991), no. 2, 229-269. MR.1111569 (92d:47029)

15. K.G. Grosse-Erdmann, Universal families and hypercyclic operators, Bull. Amer. Math. Soc. 36 (1999), no. 3, 345-381. MR1685272 (2000c:47001)

16. Hypercyclic and chaotic weighted shifts, Studia Math. 139 (2000), 47-68. MR.1763044 (2001f:47051)

17. , Recent developments in hypercyclicity, Rev. R. Acad. Cien. Serie A Mat. RACSAM 97 (2003), 273-286. MR2068180(2005c:47010)

18. G. Herzog and C. Schmoeger, On operators $T$ such that $f(T)$ is hypercyclic, Studia Math. 108 (1994), no. 3, 209-216. MR1259277 (95f:47031)

19. C. Kitai, Invariant closed sets for linear operators, Ph.D. thesis, University of Toronto, 1982.

20. G.R. MacLane, Sequences of derivatives and normal families, J. Analyse Math. 2 (1952), 72-87. MR0053231(14:741d)

21. F. Martínez-Giménez and A. Peris, Chaos for backward shift operators, Internat. J. Bifur. Chaos Appl. Sci. Engrg. 12 (2002), no. 8, 1703-1715. MR.1927407(2003h:47056)

22. R. Meise and D. Vogt, Introduction to functional analysis, Oxford University Press, OxfordNew York, 1997. MR1483073 (98g:46001)

23. A. Peris, Hypercyclicity criteria and the Mittag-Leffler theorem, Bull. Soc. Roy. Sci. Liege 70 (2001), no. 4-6, 365-371. MR 1904062 (2003c:47012)

24. S. Rolewicz, On orbits of elements, Studia Math. 32 (1969), 17-22. MR0241956 (39:3292) 
25. H.N. Salas, Hypercyclic weighted shifts, Trans. Amer. Math. Soc. 347 (1995), no. 3, 993-1004. MR 1249890 (95e:47042)

26. J.H. Shapiro, Simple connectivity and linear chaos, Rend. Circ. Mat. Palermo (2) Suppl. (1998), no. 56, 27-48, International Workshop on Operator Theory (Cefalu, 1997). MR.1710820 (2001b:47051)

27. A. Wilanski, Summability through functional analysis, North-Holland, Amsterdam, 1984. MR.0738632 (85d:40006)

28. A.H. Zemanian, Generalized integral transformations, Interscience Publishers, New York, 1968. MR0423007 (54:10991)

Departamento de Matemática Aplicada, E.T.S.I. Agrónomos, Universidad Politécnica de Valencia, Camino Vera s/n, E-46022 Valencia, Spain

E-mail address: fmarting@mat.upv.es 